\title{
AUTOMEDICAÇÃO NA COMUNIDADE: UM PROBLEMA DE SAÚDE PÚBLICA
}

\author{
Odete Amaral \\ Escola Superior de Saúde de Viseu \\ Instituto Politécnico de Viseu. Portugal \\ mopamaral@gmail.com \\ Nélio Veiga \\ Centre for Interdisciplinary Research in Health (CIIS) \\ Universidade Católica Portuguesa, Portugal \\ Paula Nelas \\ Escola Superior de Saúde de Viseu \\ Instituto Politécnico de Viseu. Portugal \\ Emília Coutinho \\ Escola Superior de Saúde de Viseu \\ Instituto Politécnico de Viseu. Portugal \\ Cláudia Chaves \\ Escola Superior de Saúde de Viseu \\ Instituto Politécnico de Viseu. Portugal
}

\section{RESUMO}

Introdução: A automedicação é um importante problema de saúde pública e constitui um desafio em diversos países europeus, designadamente em Portugal. Os objetivos deste estudo foram estimar a prevalência de automedicação numa amostra de adultos portugueses da Região Centro e Norte de Portugal e identificar fatores sociodemográficos e de saúde associados à automedicação. Participantes e métodos: Estudo transversal analítico. A amostra ficou constituída 197 indivíduos da comunidade, região centro e norte de Portugal, com uma média de idades de 38,26 $\pm 14,20$ anos e maioritariamente do género feminino $(65,0 \%)$. Os dados foram recolhidos através da aplicação de um questionário, composto por questões de caracterização sociodemográfica, de contexto de saúde e questões referentes à automedicação. Resultados: No total da amostra a prevalência de automedicação ao longo da vida foi de $74,1 \%$ e nos últimos 6 meses foi de $59,9 \%$. A automedicação ao longo da vida associou-se significativamente com a área de residência urbana $(p=0,018)$. A automedicação nos últimos 6 meses relacionou-se positivamente com a idade $\leq 25$ anos ( $O R=3,69$; IC95\% 1,04-12,14) e negativamente com a área de residência (rural OR=0,36; IC95\% 0,15-0,84). Conclusões: Observámos elevadas prevalências de automedicação ao longo da vida e nos últimos 


\section{AUTOMEDICAÇÃO NA COMUNIDADE: UM PROBLEMA DE SAÚDE PÚBLICA}

6 meses na comunidade norte e centro de Portugal. A automedicação associou-se com variáveis sociodemográficas e de saúde. Os resultados do presente estudo criam evidência para o planeamento de intervenções no âmbito do controlo da automedicação na comunidade.

Palavras-chave: adulto; automedicação; epidemiologia

\section{ABSTRACT}

Self-medication in the community: a public health issue. Background: Self-medication is an important public health issue and a challenge in several European countries, particularly in Portugal. The objectives of this study were to estimate the prevalence of self-medication in a sample of Portuguese adults in the Central and Northern Region of Portugal and to identify sociodemographic and health factors associated with self-medication. Participants and Methods: A cross-sectional analytical study was performed. The sample consisted of 197 individuals from the community, central and northern Portugal, with a mean age of $38.26 \pm 14.20$ years and mostly female $(65.0 \%)$. The data collection was accomplished through the application of a questionnaire, composed of questions of sociodemographic characterization, health context and issues related to selfmedication. Results: In the total sample, the prevalence of self-medication throughout life was $74.1 \%$ and in the last 6 months it was $59.9 \%$. Lifelong self-medication was significantly associated with urban residence $(p=0.018)$. Self-medication in the last 6 months was positively correlated with age $\leq 25$ years $(O R=3.69,95 \% \mathrm{Cl} 1.04-12.14)$ and negatively with the residential area (rural $O R=$ $0.36,95 \% \mathrm{Cl}, 0.15-0.84)$. Conclusions: We observed a high prevalence of self-medication throughout the life and in the last 6 months among the northern and central communities of Portugal. Self-medication was associated with sociodemographic and health variables. The results of the present study provide evidence for the planning of interventions in the control of selfmedication in the community.

Keywords: adult; self-medication; epidemiology

\section{INTRODUÇÃO}

A automedicação é um problema em franco crescimento em Portugal, sendo uma prática que pode acarretar riscos na saúde do indivíduo. A Organização Mundial de Saúde define automedicação como a seleção e 0 uso de medicamentos por pessoas para tratar doenças autodiagnosticadas ou sintomas e é entendida como um dos elementos do autocuidado (OMS, 1998). Em Portugal, o despacho n-17690/2007 de 23 de julho, demarca automedicação como a utilização de medicamentos não sujeitos a receita médica (MNSRM) de forma responsável, sempre que se destine ao alivio e tratamento de queixas de saúde passageiras e sem gravidade, com a assistência ou aconselhamento opcional de um profissional de saúde (Ministério da Saúde, 2007). Os MNSRM consistem em medicamentos que podem ser dispensados ao utente sem necessidade de prescrição médica, sendo vendidos em farmácias e em locais de venda autorizados para 0 efeito. Contudo, para uma utilização eficaz e eficiente da automedicação a comunidade deve compreender que estes MNSRM também apresentam riscos, dado que a automedicação pode levar o consumidor a uma dependência, atraso no diagnóstico clínico, existindo ainda riscos associados ao mau uso e à interação medicamentosa (Barros \& Nunes, 2011). No ano de 2007, em Portugal, em conformidade com um estudo da Sociedade Portuguesa de Gastrenterologia, cerca de 5\% dos doentes hospitalizados (que acabaram por morrer) por complicações do aparelho gastrointestinal, estas eram causadas pelo consumo de anti-inflamatórios não esteroides (Barros \& Nunes, 2011). 0 baixo nível de literacia em saúde da população, o não cumprimento da obrigatoriedade da receita médica e a falta de informação da população em geral, justificam a preocupação com a qualidade da automedicação praticada no mundo, principalmente tendo em conta que, em países desenvolvidos, o número de medica- 
mentos de venda livre tem crescido nos últimos tempos, assim como a disponibilidade desses medicamentos em estabelecimentos não farmacêuticos, o que favorece a automedicação (Pereira, 2009). Perante este problema e perante as competências atribuídas aos enfermeiros, estes possuem um papel determinante no empowerment da comunidade sobre os riscos da automedicação e na identificação dos sinais do uso indiscriminado de medicamentos. Ou seja, o Enfermeiro possui um papel fundamental na educação e orientação dos utentes/famílias e comunidades para a prática de uma automedicação responsável, indicando quais os riscos quando a mesma é realizada de modo não responsável (Ricardo, 2011).

Estudos realizados em Portugal mostram prevalências da automedicação muito diferentes. Um estudo realizado em meio urbano, recorrendo a uma amostra de 4135 utentes de 11 farmácias comunitárias em Lisboa e 15 farmácias no Porto revelou uma prevalência da automedicação de 26,2\% (Martins, Miranda, Mendes, Soares, Ferreira, \& Nogueira, 2002). Após regressão logística identificaram como determinantes da automedicação o maior nível de escolaridade, a ocupação (estudantes e empregados versus reformados, desempregados ou domésticas), utilização de um serviço público de saúde e maior tempo de espera por consulta médica (uma semana a um mês) (Martins, Miranda, Mendes, Soares, Ferreira, \& Nogueira, 2002). 0 mesmo estudo revelou, ainda, que metade dos inquiridos adquirir medicamentos para automedicação por conselho do farmacêutico, $30 \%$ por iniciativa própria, $18 \%$ por sugestão de amigos ou familiares e cerca de $1 \%$ por recomendação do enfermeiro (Martins, Miranda, Mendes, Soares, Ferreira, \& Nogueira, 2002). Outro estudo realizado em zonas rurais de Portugal com uma amostra de 2879 utentes em 35 farmácias mostrou uma prevalência da automedicação de $21,5 \%$, sendo o farmacêutico que mais influenciou a seleção do medicamento adquirido pelos utentes (42\%) e um dos fatores preditores da automedicação foi o tempo de espera por consulta (Melo, Madureira, Ferreira, Mendes, Miranda, \& Martins, 2006). Em Bragança, um estudo realizado com uma amostra de 400 utentes de sete farmácias e dois locais de venda de MNSRM, revelaram uma prevalência da automedicação de $91,3 \%$ e os utentes consideraram o farmacêutico como a 'fonte' de informação mais credível sobre automedicação (Martins, Couto, Ribeiro, \& Fernandes, 2011). 0 mesmo estudo revelou diferenças significativas entre os géneros no que diz respeito à prática da automedicação para 0 alívio de cólicas $(p=0,000)$ e dores musculares $(p=0,008)$ (Martins, Couto, Ribeiro, \& Fernandes, 2011). Em 2014, um estudo realizado na região centro de Portugal com uma amostra de 182 indivíduos indicou uma prevalência da automedicação ao longo da vida de $85,7 \%$ e nos últimos 6 meses de 85,9\%. A maioria da amostra $(50,3 \%)$ referiu praticar automedicação por iniciativa própria, sendo os medicamentos mais utilizados os analgésicos (78,8\%) e os anti-inflamatórios (54,5\%) (Amaral et al., 2014). 0 local de aquisição mais frequente dos medicamentos foi a farmácia $(82,5 \%)$ e, grande parte dos indivíduos $(92,3 \%)$ afirma ter conhecimento dos riscos dos medicamentos. A prática da automedicação associou-se com a idade ( $\leq 25$ anos $0 R=1,28$; IC95\% 1,16-1,41) e com 0 agregado familiar com filhos (OR=4,77; IC95\% 1,94-11,71) (Amaral et al., 2014). Noutro estudo, realizado no concelho de Chaves com uma amostra de 227 utentes de farmácias comunitárias verificaram que os medicamentos mais utilizados pelos inquiridos para a prática da automedicação foram os analgésicos e os anti-inflamatórios, constatando que $80,4 \%$ dos inquiridos já praticaram automedicação (Alves, 2012). Em Lisboa, numa amostra de 138 estudantes do Mestrado Integrado em Ciências Farmacêuticas da Universidade Lusófona de Humanidades e Tecnologias revelou uma prevalência de automedicação de 95,7\%, sendo os principais sintomas que levaram ao recurso da automedicação: os estados gripais e constipações $(14,2 \%)$, as dores menstruais $(11,1 \%)$, a tosse/rouquidão $(10,2 \%)$, cefaleias $(9,5 \%)$, febre $(8,6 \%)$, rinorreia e congestão nasal $(6,7 \%)$ e a rinite alérgica $(5,1 \%)$ (Narciso, 2013). Os medicamentos mais usados foram os anti-inflamatórios não esteroides 


\section{AUTOMEDICAÇÃO NA COMUNIDADE: UM PROBLEMA DE SAÚDE PÚBLICA}

(19,4\%), os analgésicos e antipiréticos (19,2\%), os descongestionantes nasais (12,8\%), os antitússicos e expetorantes (11,8\%), os anti-histamínicos $(9,5 \%)$ e as vitaminas e sais minerais $(7,9 \%)$ (Narciso, 2013).

Assim, dada a importância deste problema, definimos como objetivos do presente estudo estimar a prevalência de automedicação numa amostra de portugueses da Região Centro e Norte de Portugal e identificar fatores sociodemográficos e de saúde associados com a automedicação na amostra referida.

\section{MÉTODO}

\section{Participantes}

Realizámos um estudo transversal analítico. A amostra ficou constituída por 197 indivíduos da comunidade, da região centro e norte de Portugal, com uma média de idades de 38,26ะ14,20 anos (idades compreendidas entre os 18 e os 78 anos), sendo a maior percentagem do género feminino (65,0\% vs. $35,0 \%)$; mais de metade dos indivíduos é casado ou vive em união de facto $(50,3 \%)$; $41,3 \%$ reside na aldeia; $53,1 \%$ possui o ensino superior e $65,4 \%$ encontram-se empregados.

\section{Instrumento}

Os dados foram recolhidos através de um questionário autoaplicado, constituído por questões referentes à caracterização sociodemográfica, de saúde e por questões alusivas à automedicação. Para avaliar a automedicação ao longo da vida recorreu-se à questão 'alguma vez recorreu à automedicação?' e para avaliar nos últimos 6 meses questionou-se 'nos últimos 6 meses recorreu à automedicação?'. Após a colheita de dados os dados foram introduzidos e analisados no programa SPSS versão 24.0. Recorremos à estatística descritiva para determinar as frequências absolutas e percentuais, às médias e desvio padrão. As prevalências foram expressas em percentagens. Para comparação de proporções utilizou-se o teste qui quadrado com correção de Yates e respetivos intervalos de confiança a 95\% (IC95\%). A magnitude de associação entre um fator e a doença foi estimada através do cálculo do Odds Ratios (OR), com os respetivos intervalos de confiança a 95\%.

\section{RESULTADOS}

\section{Prevalência de automedicação}

A prevalência de automedicação ao longo da vida foi de 74,1\% e nos últimos 6 meses de 59,9\%. A prevalência da automedicação ao longo da vida nas mulheres foi de $65,1 \%$ e no sexo masculino de $34,9 \%$, sem diferenças significativas $(p=0,96)$ e a percentagem da automedicação nos últimos 6 meses também foi maior no sexo feminino sem diferenças significativas ( $63,6 \%$ vs. $36,4 \%$; $p=0,37)$ (Tabela 1). 
Tabela 1 - Prevalência de automedicação ao longo da vida e nos últimos 6 meses

\begin{tabular}{|c|c|c|c|c|c|c|c|}
\hline & \multicolumn{2}{|c|}{ Feminino } & \multicolumn{2}{|c|}{ Masculino } & \multirow[b]{2}{*}{$p$} & \multicolumn{2}{|c|}{ Total } \\
\hline & $\mathrm{n}$ & $\%$ & $\mathrm{n}$ & $\%$ & & $\mathrm{n}$ & $\%$ \\
\hline $\begin{array}{l}\text { Automedicação (alguma } \\
\text { vez) }\end{array}$ & $\begin{array}{c}12 \\
8\end{array}$ & 65,0 & 69 & 35,0 & & $\begin{array}{c}19 \\
7\end{array}$ & 100,0 \\
\hline Sim & 95 & 65,1 & 51 & 34,9 & 0,96 & $\begin{array}{c}14 \\
6\end{array}$ & 74,1 \\
\hline Não & 33 & 64,7 & 18 & 35,3 & & 51 & 25,9 \\
\hline $\begin{array}{l}\text { Automedicação últimos } 6 \\
\text { meses }\end{array}$ & 95 & 65,1 & 51 & 34,9 & & $\begin{array}{c}14 \\
6\end{array}$ & 100,0 \\
\hline Sim & 75 & 63,6 & 43 & 36,4 & 0,37 & $\begin{array}{c}11 \\
8\end{array}$ & 59,9 \\
\hline Não & 20 & 71,4 & 8 & 26,8 & & 28 & 14,1 \\
\hline
\end{tabular}

\section{Caracterização da automedicação}

Das pessoas que referiram recorrer à automedicação, 4,1\% fê-lo uma vez por semana ou mais; 4,8\% uma vez por quinzena; $19,9 \%$ uma vez por mês; $16,4 \%$ uma vez por cada 3 meses e $54,8 \%$ referiu fazer com menos frequência. Quanto à duração da toma do medicamento, a maioria dos indivíduos referiu 1 a 2 dias (51,8\%); 24,5\% 3 a 4 dias e 6,1\% mencionou uma duração de 5 ou mais dias. Os grupos de medicamentos mais utilizados na automedicação foram os analgésicos (56,9\%); os anti-inflamatórios (43,1\%); os antipiréticos (18,8\%); as vitaminas $(5,6 \%)$; os antibióticos $(2,5 \%)$; os antidepressivos $(0,5 \%)$ e os anti-histamínicos $(0,5 \%)$.

Os problemas de saúde que os participantes referiram apresentar quando recorreram à automedicação foram dores $(48,7 \%)$, gripe $(28,9 \%)$, constipação $(27,4 \%)$, febre $(19,8 \%)$, infeções $(14,7 \%)$, tosse $(11,2 \%)$, problemas de sono $(4,1 \%)$ e em menor percentagem referiram falta de apetite, tristeza, astenia, ansiedade e enxaqueca, todas as condições com igual percentagem $(0,5 \%)$. A maioria da amostra referiu adquirir os medicamentos para a automedicação na farmácia $(66,0 \%)$; $20,3 \%$ afirmou que usou as sobras de medicamentos anteriormente receitados e $4,6 \%$ referiu que um familiar ou amigo partilhou-o consigo. A maioria mencionou que procurou informação sobre 0 medicamento antes de 0 tomar $(71,9 \%)$; e procurou essa informação na bula $(56,60 \%)$, consultando 0 farmacêutico $(50,0 \%)$, o enfermeiro $(16,04 \%)$ e $9,4 \%$ dos indivíduos procurou a informação através de um amigo ou familiar, contudo apenas $58,9 \%$ dos indivíduos referiu que percebeu a informação recebida.

Para além da automedicação, $31,6 \%$ da amostra referiu que já recorreu a outro tipo de produtos para resolver os seus problemas de saúde, designadamente $74,6 \%$ referenciou já ter recorrido a chás; $27,1 \%$ a mesinhas caseiras; $11,9 \%$ a suplementos alimentares; $10,2 \%$ a produtos naturais e 8,5\% à acupunctura.

\section{Fatores associados com a automedicação}

De acordo com a Tabela 2, a automedicação ao longo da vida associou-se com a área de residência ( $p=0,018) ; 45,9 \%$ dos indivíduos que vivem na zona rural e $54,1 \%$ dos que vivem na zona urbana referiram que alguma vez já praticaram automedicação. As restantes variáveis sociodemográficas não se associaram significativamente com a automedicação ao longo da vida. 


\section{AUTOMEDICAÇÃO NA COMUNIDADE: UM PROBLEMA DE SAÚDE PÚBLICA}

Tabela 2 - Fatores sociodemográficos associados à automedicação ao longo da vida

\begin{tabular}{|c|c|c|}
\hline \multicolumn{3}{|c|}{ Alguma vez praticou automedicação } \\
\hline Sim & Não & Total \\
\hline$n(\%)$ & $\mathrm{n}(\%)$ & $\mathrm{n}(\%)$ \\
\hline
\end{tabular}

\begin{tabular}{|c|c|c|c|}
\hline Sexo & & & \\
\hline Feminino & $95(65,1 \%)$ & $\begin{array}{c}33 \\
(64,7 \%)\end{array}$ & $\begin{array}{c}128 \\
(65,0 \%)\end{array}$ \\
\hline Masculino & $51(34,9 \%)$ & $\begin{array}{c}18 \\
(35,3 \%)\end{array}$ & $\begin{array}{c}69 \\
(35,0 \%)\end{array}$ \\
\hline$p$ & 0,93 & & \\
\hline Idade & & & \\
\hline$\leq 25$ anos & $44(30,1 \%)$ & $\begin{array}{c}14 \\
(27,5 \%)\end{array}$ & $\begin{array}{c}58 \\
(29,4 \%)\end{array}$ \\
\hline $26-55$ anos & $84(57,5 \%)$ & $\begin{array}{c}29 \\
(25,7 \%)\end{array}$ & $\begin{array}{c}113 \\
(57,4 \%)\end{array}$ \\
\hline$\geq 56$ anos & $18(12,3 \%)$ & $8(15,7 \%)$ & $\begin{array}{c}26 \\
(13,2 \%)\end{array}$ \\
\hline$p$ & 0,81 & & \\
\hline Estado civil & & & \\
\hline Casado & $74(50,7 \%)$ & $\begin{array}{c}25 \\
(49,0 \%)\end{array}$ & $\begin{array}{c}99 \\
(50,3 \%)\end{array}$ \\
\hline Outro & $72(49,3 \%)$ & $\begin{array}{c}26 \\
(51,0 \%)\end{array}$ & $\begin{array}{c}98 \\
(49,7 \%)\end{array}$ \\
\hline$p$ & 0,84 & & \\
\hline Habilitações literárias & & & \\
\hline $1^{\circ}$ ciclo - secundário & $67(46,2 \%)$ & $\begin{array}{c}25 \\
(49,0 \%)\end{array}$ & $\begin{array}{c}92 \\
(46,9 \%)\end{array}$ \\
\hline Ensino Superior & $78(53,8 \%)$ & $\begin{array}{c}26 \\
(51,0 \%)\end{array}$ & $\begin{array}{c}104 \\
(53,1 \%)\end{array}$ \\
\hline$p$ & 0,72 & & \\
\hline Área de residência & & & \\
\hline Rural & $67(45,9 \%)$ & $\begin{array}{c}18 \\
(35,3 \%)\end{array}$ & $\begin{array}{c}85 \\
(43,1 \%)\end{array}$ \\
\hline Urbana & $79(54,1 \%)$ & $\begin{array}{c}33 \\
(64,7 \%)\end{array}$ & $\begin{array}{c}112 \\
(56,9 \%)\end{array}$ \\
\hline$p$ & 0,018 & & \\
\hline Situação laboral & & & \\
\hline Empregado & $92(64,8 \%)$ & $\begin{array}{c}33 \\
(67,3 \%)\end{array}$ & $\begin{array}{c}125 \\
(65,4 \%)\end{array}$ \\
\hline Outro & $50(35,2 \%)$ & $\begin{array}{c}16 \\
(32,7 \%)\end{array}$ & $\begin{array}{c}66 \\
(34,6 \%)\end{array}$ \\
\hline$p$ & 0,75 & & \\
\hline
\end{tabular}

Dos fatores sociodemográficos, pela análise da Tabela 3, verificamos que a automedicação nos últimos 6 meses se associou positivamente com a idade $\leq 25$ anos (OR=3,69; IC95\% 1,04-12,14) e negativamente com a área de residência rural (OR=0,36; IC95\% 0,15-0,84). 
Tabela 3 - Fatores sociodemográficos associados à automedicação nos últimos 6 meses

\begin{tabular}{|c|c|}
\hline & $\begin{array}{l}\text { Automedicação } \\
\text { últimos } 6 \text { meses }\end{array}$ \\
\hline & OR (IC95\%) \\
\hline \multicolumn{2}{|l|}{ Sexo } \\
\hline Feminino & $0,66(0,27-1,63)$ \\
\hline Masculino & $1 *$ \\
\hline \multicolumn{2}{|l|}{ Idade } \\
\hline$\leq 25$ anos & $3,69(1,04-12,14)$ \\
\hline 26-55 anos & $2,48(0,84-7,30)$ \\
\hline$\geq 56$ anos & $1 *$ \\
\hline \multicolumn{2}{|l|}{ Área de residência } \\
\hline Rural & $0,36(0,15-0,84)$ \\
\hline Urbana & $1 *$ \\
\hline \multicolumn{2}{|l|}{ Estado civil } \\
\hline Casado & $1,12(0,49-2,50)$ \\
\hline Outro & $1 *$ \\
\hline \multicolumn{2}{|c|}{ Habilitações literárias } \\
\hline $\begin{array}{l}1^{\circ} \quad \text { ciclo } \\
\text { secundário }\end{array}$ & $1,06(0,47-2,39)$ \\
\hline Ensino Superior & $1 *$ \\
\hline \multicolumn{2}{|l|}{ Situação laboral } \\
\hline Empregado & $0,70(0,28-1,72)$ \\
\hline Outro & $1 *$ \\
\hline
\end{tabular}

Quantos aos hábitos de vida, e de acordo com a Tabela 4, os indivíduos que indicaram praticar exercício físico apresentaram um risco superior de terem praticado automedicação nos últimos 6 meses (OR=2,55; IC95\% 1,09-5,95). Quando cruzámos as variáveis 'perceção da saúde em geral' com a automedicação não se encontrou associação com diferenças significativas. Os indivíduos com familiares que apresentam doenças crónicas apresentam um risco superior de terem praticado automedicação nos últimos 6 meses (OR=3,09; IC95\% 1,10-8,68). 


\section{AUTOMEDICAÇÃO NA COMUNIDADE: UM PROBLEMA DE SAÚDE PÚBLICA}

Tabela 4 - Hábitos de vida e variáveis clínicas associadas à automedicação

\begin{tabular}{|c|c|c|}
\hline & $\begin{array}{l}\text { Automedicação } \\
\text { (ao longo da vida) }\end{array}$ & $\begin{array}{c}\text { Automedicação } \\
\text { (últimos } 6 \\
\text { meses) }\end{array}$ \\
\hline & OR (IC95\%) & OR (IC95\%) \\
\hline \multicolumn{3}{|c|}{ Consumo de bebidas alcoólicas } \\
\hline Sim & $1,78(0,69-4,58)$ & $\begin{array}{c}1,16(0,40- \\
3,37)\end{array}$ \\
\hline Não & $1^{*}$ & $1^{*}$ \\
\hline \multicolumn{3}{|l|}{ Fumador } \\
\hline Sim & $0,93(0,45-1,91)$ & $\begin{array}{c}1,49(0,56- \\
3,98)\end{array}$ \\
\hline Não & $1^{*}$ & $1^{*}$ \\
\hline \multicolumn{3}{|l|}{ Exercício físico } \\
\hline Sim & $0,97(0,51-1,86)$ & $\begin{array}{l}2,55(1,09- \\
5,95)\end{array}$ \\
\hline Não & $1^{*}$ & $1^{*}$ \\
\hline \multicolumn{3}{|l|}{ Saúde em geral } \\
\hline $\begin{array}{l}\text { Ótima/Muito boa/ } \\
\text { boa } \\
\text { Razoável / má }\end{array}$ & $\begin{array}{c}1,38(0,65-2,92) \\
1^{*}\end{array}$ & $\begin{array}{c}0,41(0,11- \\
1,46) \\
1^{*}\end{array}$ \\
\hline \multicolumn{3}{|c|}{ Habitualmente toma medicação } \\
\hline Sim & $\begin{array}{c}0,59(0,31-1,13) \\
*\end{array}$ & $\begin{array}{c}0,89(0,38- \\
2,10)\end{array}$ \\
\hline Não & $1^{*}$ & $1^{*}$ \\
\hline \multicolumn{3}{|c|}{ Doenças crónicas na família } \\
\hline Sim & $1,53(0,75-3,14)$ & $\begin{array}{c}3,09(1,10- \\
8,68)\end{array}$ \\
\hline Não & $1^{*}$ & $1^{*}$ \\
\hline
\end{tabular}

\section{DISCUSSÃO}

A automedicação é uma prática a que as pessoas recorrem frequentemente. No presente estudo e na maioria dos estudos realizados em Portugal em adultos, considerou-se automedicação como a utilização de qualquer medicamento sem prescrição, independentemente da classificação quanto à dispensa.

A prevalência da automedicação no presente estudo foi de $74,1 \%$ ao longo da vida e nos últimos 6 meses de 59,9\%, sem diferenças significativas entre os sexos. Estes resultados corroboram os encontrados por Amaral et al. (2014) que, no seu estudo encontrou uma prevalência ao longo da vida de automedicação de 85,7\%. Contudo, nos diferentes estudos encontrados a prevalência da automedicação mostra-se muito variável, estudos realizados em Portugal com adultos revelaram prevalências de automedicação a variar entre 21\% e 96\% (Amaral, et. al., 2014; Narciso, 2013; Alves, 2012; Martins, Couto, Ribeiro, \& Fernandes, 2011; Melo, Madureira, Ferreira, Mendes, Miranda, \& Martins, 2006; Martins, Miranda, Mendes, Soares, Ferreira, \& Nogueira, 2002). Apurouse que os grupos de medicamentos mais utilizados na automedicação foram os analgésicos 
(56,9\%); os anti-inflamatórios (43,1\%); os antipiréticos (18,8\%); as vitaminas $(5,6 \%)$; os antibióticos $(2,5 \%)$; os antidepressivos ( $0,5 \%)$ e os anti-histamínicos (0,5\%), o que está em conformidade com outros estudos sobretudo no que diz respeito aos analgésicos, antipiréticos e os anti-inflamatórios (Amaral et al., 2014; Narciso, 2013; Matos, Pena, Parreira, Santos, \& Coura-Vital, 2018). Um estudo realizado no Brasil com 270 indivíduos (181 adolescentes e 89 adultos), com idade média de $23,1 \pm 10,8$, mostrou uma elevada proporção de indivíduos que se automedicavam $69,3 \%$ (IC95\%: 63,6-74,6) e os medicamentos mais utilizados foram os analgésicos ( $n=123 ; 65,8 \%$ ), antigripais $(21,4 \%)$ e os anti-inflamatórios (18,7\%) (Matos, Pena, Parreira, Santos, \& Coura-Vital, 2018). No presente estudo ressalvo que $2,5 \%$ da amostra referiu automedicar-se com antibióticos, mas comprar antibiótico na farmácia necessita de receita em Portugal. Perante estes dados possivelmente as pessoas utilizam sobras de antibióticos prescritos anteriormente ou a farmácia vende 0 medicamento sem receita médica. Na presente investigação, a maioria da amostra referiu que adquiriu os medicamentos para automedicação na farmácia (66,0\%); 20,3\% afirmou que usou as sobras de medicamentos anteriormente receitados e 4,6\% referiu que um familiar ou amigo partilhou-0 consigo. Estes resultados corroboram os encontrados noutros estudos (Martins, Miranda, Mendes, Soares, Ferreira, \& Nogueira, 2002; Amaral et al., 2014; Cruz, Caramona, \& Guerreiro, 2015) que revelam que o local de aquisição mais frequente dos medicamentos para a automedicação mencionado foi a farmácia. Os problemas de saúde existentes para os indivíduos recorrerem à automedicação foram dores $(48,7 \%)$, gripe $(28,9 \%)$, constipação $(27,4 \%)$, febre $(19,8 \%)$, infeções $(14,7 \%)$, tosse $(11,2 \%)$, problemas de sono $(4,1 \%)$ e em menor percentagem referiram a falta de apetite, tristeza, astenia, ansiedade e enxaqueca. Um estudo realizado em Portugal com estudantes concluiu que os principais sintomas que levaram ao recurso da automedicação foram os estados gripais e constipações $(14,2 \%)$, as dores menstruais $(11,1 \%)$, a tosse/rouquidão $(10,2 \%)$, cefaleias $(9,5 \%)$, febre $(8,6 \%)$, rinorreia e congestão nasal $(6,7 \%)$ e a rinite alérgica $(5,1 \%)$ (Narciso, 2013). Outro estudo realizado no Brasil mostrou que o sintoma responsável pela maior parte dos casos de automedicação foi a cefaleia/febre $(67,9 \%)$, seguido dos estados gripais (32,6\%) (Matos, Pena, Parreira, Santos, \& Coura-Vital, 2018).

Em relação aos fatores associados com a prática da automedicação, a presente investigação identificou como fatores de risco a idade $\leq 25$ anos, o exercício físico e a presença de doenças crónicas na família e como fatores de proteção viver no meio rural. Em 2018, um estudo realizado no Brasil identificou como fatores associados à automedicação a influencia da publicidade $(R P=1,2$; IC95\%=1,1-1,4); a prática de indicar algum medicamento para outras pessoas ( $R P=1,4$; IC95\%=1,1-1,6); ter realizado a última consulta médica há mais de um mês da data da entrevista aumenta em 1,5 vezes a prevalência de se automedicar ( $R P=1,5 ; \quad I C 95 \%=1,1-2,1)$ e 0 estado de saúde autorrelatado de 'muito bom/bom' diminui prevalência de automedicação ( $R P=0,8$; IC95\%=0,6-0,9) (Matos, Pena, Parreira, Santos, \& Coura-Vital, 2018).

\section{CONCLUSÕES}

Verificámos que a automedicação constitui um fenómeno comum. Os principais grupos de medicamentos utilizados foram os analgésicos, anti-inflamatórios e antipiréticos. Em relação aos fatores sociodemográficos e de saúde associados com a automedicação, concluiu-se que a automedicação ao longo da vida associou-se com a área de residência e a automedicação nos últimos 6 meses associou-se positivamente com a idade $\leq 25$ anos e negativamente com a área de residência rural. Este estudo ajudou na identificação de situações com necessidade de intervenção por forma a aumentar a literacia em saúde e tornar a automedicação uma prática mais segura. Os profissionais de saúde têm um papel fundamental na consciencialização das comunidades. 


\section{AUTOMEDICAÇÃO NA COMUNIDADE: UM PROBLEMA DE SAÚDE PÚBLICA}

\section{AGRADECIMENTOS}

Agradecimento ao Fundo Social Europeu, ao Programa Operacional Portugal 2020, Centro 2020 e à Fundação para a Ciência e Tecnologia do Governo Português, instituições públicas que se constituem como fontes de financiamento do Projeto de Investigação Medelderly (FCT / MCTES), Portugal 2020 e do Centro 2020 (SAICT-POL / 23585/2016).

\section{REFERÊNCIAS}

Alves, M.R. (2012). Frequência de automedicação em residentes do concelho de Chaves. Tese de mestrado. Universidade Fernando Pessoa, 2012.

Amaral 0, Lages, A., Sousa, L., Almeida, L., Santos, J., Dias, M., et al. (2014). Automedicação em Jovens e Adultos da Região Centro de Portugal. Millenium, 47, 97109.

Barros, P.P., \& Nunes, L.C. (2011). A liberalização dos Medicamentos Não Sujeitos a Receita Médica. In: Barros PP, Nunes LC, editors. 10 Anos de Política do Medicamento em Portugal. 2011. 101-50.

Cruz, P.S., Caramona, M., \& Guerreiro, M.P. (2015). Uma reflexão sobre a automedicação e medicamentos não sujeitos a receita médica em Portugal. Revista Portuguesa de Farmacoterapia, $7(2), 83-90$.

Despacho n.. 17690/2007, de 23 de julho. Diário da República, 2. a série, n.ำ154, de 10 de agosto de 2007. Acedido em http://www.infarmed.pt/documents/15786/1065790/011D1_Desp_17690_2007.pdf

Martins, A.P., Miranda, A.D.C., Mendes, Z., Soares, M.A., Ferreira, P., \& Nogueira, A. (2002). Selfmedication in a Portuguese urban population: a prevalence study. Pharmacoepidemiol Drug Safety, 11(5), 409-14.

Martins, D., Couto, S.M.P., Ribeiro, M.I.B.R., \& Fernandes, A.J.G.F. Prevalência da automedicação na região de Bragança: a perspetiva do consumidor e do farmacêutico. Egitania Sciencia, 8, 199215.

Matos, J.F., Pena, D.A.C., Parreira, M.P., Santos, T.C., \& Coura-Vital W. (2018). Prevalência, perfil e fatores associados à automedicação em adolescentes e servidores de uma escola pública profissionalizante. Cadernos de Saúde Coletiva, Rio de Janeiro, 26 (1), 76-83.

Melo, M.N., Madureira, B., Ferreira, A.P.N., Mendes, Z., Miranda, A.D.C., \& Martins, A.P. (2006). Prevalence of self-medication in rural areas of Portugal. Pharmacy World \& Science, 28(1):1925.

Narciso, A.P.S. (2013). Prevalência da Automedicação nos alunos do Mestrado Integrado em Ciências Farmacêuticas da Universidade Lusófona de Humanidades e Tecnologias. Tese Mestrado em Ciências Farmacêuticas. Lisboa 2013.

Pereira, D. (2009). Frequência da automedicação em farmácias comunitárias, https://bdigital.ufp.pt/dspace/bitstream/10284/1102/3/monografia.pdf

Ricardo, A. F. T. (2011). Automedicação no aluno universitário. Projeto de graduação ao grau de licenciatura em enfermagem - Universidade Fernando Pessoa. Porto, 2011.

World Health Organization (1998). The role of the pharmacist in self-care and self-medication. Report of the $4^{\text {th }}$ WHO Consultative Group on the Role of the Pharmacist. Department of Essential Drugs and Other Medicines World Health Organization. Netherlands. 